\title{
Multivariate modified Fourier expansions
}

Ben Adcock and Daan Huybrechs

\begin{abstract}
In this paper, we review recent advances in the approximation of multivariate functions using eigenfunctions of the Laplace operator subject to homogeneous Neumann boundary conditions. Such eigenfunctions are known explicitly on a variety of domains, including the $d$-variate cube, equilateral triangle and numerous other higher dimensional simplices. Practical construction of truncated expansions is achieved using a mixture of asymptotic and classical quadratures. Moreover, by exploiting the hyperbolic cross, the number of expansion coefficients need only grow mildly with dimension.

Despite converging uniformly throughout the domain, the rate of convergence of such expansions may be slow. We review two techniques to accelerate convergence. The first smoothes the function by interpolating certain derivatives of the function evaluated on the boundary of the domain. The second numerically computes a smooth, periodic extension of the function on a larger domain.
\end{abstract}

\section{Introduction}

The subject of this paper is the approximation of a multivariate function $f: \Omega \rightarrow \mathbb{R}$ in eigenfunctions of the Laplace operator subject to homogeneous Neumann boundary conditions:

$$
-\triangle \phi(x)=\mu \phi(x), \quad x \in \Omega, \quad \frac{\partial \phi}{\partial n}(x)=0, \quad x \in \partial \Omega .
$$

Ben Adcock

DAMTP, Centre for Mathematical Sciences, University of Cambridge, Wilberforce Rd, Cambridge, CB3 0WA, UK, e-mail: b.j.s.adcock@ damtp.cam.ac.uk

Daan Huybrechs

Department of Computer Science, Katholieke Universiteit Leuven, Celestijnenlaan 200A, B-3001

Leuven, Belgium, e-mail: daan.huybrechs@cs.kuleuven.be 
The one-dimensional case of (1) was proposed and studied in [16] and has led to a rapidly growing list of papers on numerical aspects of eigenfunction expansions and related topics, which we will briefly review throughout this text.

We assume that the domain $\Omega \subset \mathbb{R}^{d}$ is non-empty, bounded and simply connected with piecewise smooth boundary. The eigenvalues of (1), $\mu_{n} \geq 0, n \in \mathbb{N}$, are real, countable and have no finite limit point in $\mathbb{R}$. The corresponding eigenfunctions, $\phi_{n}(x)$, are orthogonal. Aside from the zero eigenvalue $\mu_{0}=0$ with eigenfunction $\phi_{0}(x) \equiv 1$, all eigenvalues are positive.

Let $(\cdot, \cdot)$ be the standard Euclidean inner product on $\Omega$ with associated norm $\|\cdot\|$. A function $f$ may be expanded in the infinite series

$$
f(x) \sim \sum_{n=0}^{\infty} \frac{1}{\left\|\phi_{n}\right\|^{2}} \hat{f}_{n} \phi_{n}(x)
$$

where $\hat{f}_{n}=\left(f, \phi_{n}\right)$. Standard spectral theory confirms density of the set $\left\{\phi_{n}: n \in \mathbb{N}\right\}$ in $L^{2}(\Omega)$ [11], thereby verifying convergence of this expansion in the $L^{2}(\Omega)$ norm.

In the unit interval $\Omega=(-1,1)$, trivial calculations establish that

$$
f(x) \sim \frac{1}{2} \hat{f}_{0}^{[0]}+\sum_{n=1}^{\infty}\left\{\hat{f}_{n}^{[0]} \phi_{n}^{[0]}(x)+\hat{f}_{n}^{[1]} \phi_{n}^{[1]}(x)\right\},
$$

where $\phi_{n}^{[0]}(x)=\cos n \pi x$ and $\phi_{n}^{[1]}(x)=\sin \left(n-\frac{1}{2}\right) \pi x$. Such expansion bears a striking resemblance to the classical Fourier expansion. For this reason we refer to (2) as a modified Fourier expansion. However, unlike the former, the series (3) converges uniformly on $[-1,1]$. If this series is truncated after $N$ terms, the uniform error is $\mathscr{O}\left(N^{-1}\right)$, whereas away from the endpoints the pointwise error is $\mathscr{O}\left(N^{-2}\right)$ [21]. In comparison, the truncated Fourier sum exhibits an $\mathscr{O}\left(N^{-1}\right)$ error away from the endpoints, but uniform convergence is lacking (the Gibbs phenomenon).

The improvement in convergence stems from the Neumann boundary conditions. Suppose that $\mu \neq 0$ is a Laplace-Neumann eigenvalue with corresponding eigenfunction $\phi$. Then, for $f \in H^{2}(\Omega)$, two applications of Stokes' theorem yields

$$
\begin{aligned}
(f, \phi) & =-\frac{1}{\mu}(f, \triangle \phi) \\
& =-\frac{1}{\mu} \int_{\partial \Omega} f(x) \frac{\partial \phi(x)}{\partial n} d x+\frac{1}{\mu} \int_{\partial \Omega} \frac{\partial f(x)}{\partial n} \phi(x) d x-\frac{1}{\mu}(\triangle f, \phi) .
\end{aligned}
$$

After substituting the boundary conditions, we obtain

$$
(f, \phi)=\frac{1}{\mu} \int_{\partial \Omega} \frac{\partial f(x)}{\partial n} \phi(x) d x-\frac{1}{\mu}(\triangle f, \phi) .
$$

In the univariate setting, for example, the coefficients $\hat{f}_{n}^{[i]}=\mathscr{O}\left(n^{-2}\right)$, ensuring uniform convergence of the expansion. In fact, iterating the above process, we obtain 


$$
\hat{f}_{n}^{[i]}=\sum_{r=0}^{k-1} \frac{(-1)^{n+i}\left[f^{(2 r+1)}(1)+(-1)^{i+1} f^{(2 r+1)}(-1)\right]}{\left[\left(n-\frac{i}{2}\right) \pi\right]^{2 r+2}}+\mathscr{O}\left(n^{-2 k-2}\right),
$$

for any $k \in \mathbb{N}_{+}=\mathbb{N} \backslash\{0\}$. In comparison, the classical Fourier sine coefficient

$$
\int_{-1}^{1} f(x) \sin n \pi x d x=\mathscr{O}\left(n^{-1}\right) .
$$

Non-uniform convergence of the Fourier expansion is now apparent.

Modified Fourier expansions possess great generality and relative simplicity. However, in the tensor product setting at least, they are usually cast aside in favour of spectrally convergent approximations comprised of orthogonal polynomials.

Nonetheless, such expansions enjoy a number of advantages. First, the coefficients $\hat{f}_{n}$ can be computed efficiently using a variety of numerical quadratures. The mainstay of this is the observation that the eigenfunctions $\phi_{n}$ become increasingly oscillatory for large $n$, thus facilitating the use of efficient computational schemes for highly oscillatory integrals. These enable the computation of coefficients oneby-one at a fixed cost per coefficient. In this manner, any $M$ coefficients can be computed in $\mathscr{O}(M)$ operations. This approach is also completely adaptive: increasing $M$ does not require recalculation of existing values. When looking at the computational cost, such a scheme compares favourably over alternative approaches, in particular the FFT. On the other hand, unlike in the FFT, the accuracy of each computed coefficient is not necessarily coupled to the total number of coefficients. Still, due to this adaptivity, modified Fourier expansions can successfully exploit tools such as the hyperbolic cross to greatly reduce the number of approximation coefficients. We consider this further in Section 3. For a discussion of the particular quadratures used in modified Fourier expansions, we refer the reader to $[14,16,18]$.

Modified Fourier series offer a number of benefits with regard to applications. First, they lead to considerably better conditioned matrices than polynomial-based spectral methods for differential equations $[2,3]$. Second, they allow for cheaper and faster calculation of spectra of highly oscillatory Fredholm operators [8].

Modified Fourier expansions possess at least one other virtue: Laplace-Neumann eigenfunctions are known explicitly in a number of non-tensor product domains, including the equilateral triangle [14]. We consider this further in Section 5.

Unfortunately the convergence rate of such expansions may be slow. In Section 4 we introduce two techniques to accelerate convergence.

\section{The $d$-variate cube}

Univariate modified Fourier expansions were introduced in [16]. Their extension to the $d$-variate cube $\Omega=(-1,1)^{d}$, studied in [18], is obtained by Cartesian products. If $n=\left(n_{1}, \ldots, n_{d}\right) \in \mathbb{N}^{d}$ and $i=\left(i_{1}, \ldots, i_{d}\right) \in\{0,1\}^{d}$ are multi-indices then 


$$
f(x) \sim \sum_{i \in\{0,1\}^{d}} \sum_{n \in \mathbb{N}^{d}} \hat{f}_{n}^{[i]} \phi_{n}^{[i]}(x)
$$

where $\phi_{n}^{[i]}(x)=\phi_{n_{1}}^{\left[i_{1}\right]}\left(x_{1}\right) \ldots \phi_{n_{d}}^{\left[i_{d}\right]}\left(x_{d}\right)$. Convergence of the expansion (5) has been addressed in [2]. As described, this is best studied in so-called Sobolev spaces of dominating mixed smoothness:

$$
H_{\text {mix }}^{q}(\Omega)=\left\{f \in L^{2}(\Omega): \partial_{x_{1}}^{\beta_{1}} \ldots \partial_{x_{d}}^{\beta_{d}} f \in L^{2}(\Omega), 0 \leq \beta_{1}, \ldots, \beta_{d} \leq q\right\}, \quad q \in \mathbb{N} .
$$

Observe that $H^{q d}(\Omega) \subset H_{\text {mix }}^{q}(\Omega) \subset H^{q}(\Omega)$, where $H^{q}(\Omega)$ is the $q^{\text {th }}$ classical Sobolev space. Such spaces occur in a range of applications, including sparse grids [9] and hyperbolic cross approximations [22, 23]. In relation to modified Fourier expansions, we have the following result:

Theorem 1 (Adcock [2]). The set $\left\{\phi_{n}^{[i]}: n \in \mathbb{N}^{d}, i \in\{0,1\}^{d}\right\}$ is dense and orthogonal in $H_{\text {mix }}^{1}(-1,1)^{d}$.

This theorem highlights the advantage over the Fourier basis. Uniform convergence of the expansion (5) follows immediately from the continuous embedding $H_{\text {mix }}^{1}(\Omega) \hookrightarrow C(\bar{\Omega})[2]$.

For numerical computations we must truncate the expansion (5) suitably. To do so, we include only those coefficients $n \in I_{N}$, where $I_{N} \subset \mathbb{N}^{d}$ is finite. Standard intuition leads to the full index set

$$
I_{N}=\left\{n \in \mathbb{N}^{d}: 0 \leq n_{1}, \ldots, n_{d} \leq N\right\} .
$$

Provided the function $f$ is sufficiently smooth, convergence rates of $\mathscr{O}\left(N^{-2}\right)$ and $\mathscr{O}\left(N^{-1}\right)$ are observed inside the domain and on the boundary respectively $[2,21]$. Unfortunately $\left|I_{N}\right|=\mathscr{O}\left(N^{d}\right)$, rendering such expansions expensive to construct in two or more dimensions. However, as we now consider, this figure can be significantly reduced without affecting convergence rates unduly.

\section{The hyperbolic cross}

The majority of coefficients $\hat{f}_{n}^{[i]}$ with indices $n$ in the set (6) have negligible contribution to the overall approximation. An alternative criterion to define $I_{N}$ is to include only those coefficients with absolute value greater than some tolerance $\varepsilon$.

Due to the simple tensor product setting, the coefficients $\hat{f}_{n}^{[i]}$ are $\mathscr{O}\left(n_{1}^{-2} \ldots n_{d}^{-2}\right)$ for large $n_{1}, \ldots, n_{d}$, provided $f \in H_{\text {mix }}^{2}(\Omega)$. In fact, by applying the univariate expansion (4) in each variable, we readily obtain a multivariate analogue $[2,18]$. Returning to the construction of $I_{N}$ and setting the tolerance $\varepsilon=N^{-2}$ we obtain the hyperbolic cross index set

$$
I_{N}=\left\{n \in \mathbb{N}^{d}: \bar{n}_{1} \ldots \bar{n}_{d} \leq N\right\}
$$


where $\bar{m}=\max \{m, 1\}$ for $m \in \mathbb{N}$. A simple calculation (see [13]) verifies that $\left|I_{N}\right|=$ $\mathscr{O}\left(N(\log N)^{d-1}\right)$. This figure grows much more mildly with dimension than the corresponding value of $\mathscr{O}\left(N^{d}\right)$ for the index set (6).

The application of the hyperbolic cross (7) to modified Fourier expansions was introduced in [13]. As the following theorem demonstrates, this greatly increases their effectiveness without deteriorating the convergence rate unduly:

Theorem 2 (Adcock [2]). Suppose that $f_{N}$ is the truncated modified Fourier expansion of $f$ based on (7). Then $f(x)-f_{N}(x)$ is $\mathscr{O}\left(N^{-2}(\log N)^{d-1}\right)$ for $x \in \Omega$ and $\mathscr{O}\left(N^{-1}(\log N)^{d-1}\right)$ for $x \in \partial \Omega$.

\section{Accelerating convergence}

As demonstrated, the convergence rate of modified Fourier expansion is typically slow. In this section we discuss two approaches to accelerate convergence.

\subsection{The Lanczos representation and its computation}

Consider the univariate setting. The quality of the approximation $f_{N}$ is improved if $f$ satisfies

$$
f^{(2 r+1)}( \pm 1)=0, \quad r=0, \ldots, k-1,
$$

for some $k=1,2, \ldots$ (the analogue of periodicity for modified Fourier expansions). This is manifested in a number of ways. First, recalling (4), we observe more rapid decay of the coefficients $\hat{f}_{n}^{[i]}=\mathscr{O}\left(n^{-2 k-2}\right)$. Moreover, the expansion $f_{N}$ converges to $f$ in the $H^{2 k+1}(-1,1)$ norm [3], and the pointwise convergence rate is $\mathscr{O}\left(N^{-2 k-2}\right)$ in $(-1,1)$ and $\mathscr{O}\left(N^{-2 k-1}\right)$ at the endpoints [21]. If $f$ does not satisfy (8) a standard approach is to seek a smooth function $p$ such that

$$
p^{(2 r+1)}( \pm 1)=f^{(2 r+1)}( \pm 1), \quad r=0, \ldots, k-1,
$$

and decompose $f$ as $(f-p)+p$. This is referred to as the Lanczos representation [20]. Since $f-p$ obeys (8), the new approximation defined by $g_{N}=f_{N}-p_{N}+p$ converges at the faster rates prescribed above. Usually the function $p$ is chosen to be a polynomial of degree $2 k$. In view of this fact, this process is often referred to as polynomial subtraction [19].

Unfortunately, this process requires exact derivatives. Such values (or functions of $(d-1)$ variables when $d \geq 2)$ are unknown in general. However, if approximated to sufficient accuracy, the convergence rate of $g_{N}$ need not deteriorate. One approach to accomplish this is Eckhoff's method [10].

The approximation $g_{N}$ (a linear combination of a finite modified Fourier sum and a polynomial) with $p$ satisfying (9) also satisfies ${\widehat{g_{N}}}_{n}^{[i]}=\hat{f}_{n}^{[i]}+\mathscr{O}\left(n^{-2 k-2}\right)$. To avoid 
the use of derivatives, we construct a new function $g_{N}$ that satisfies this condition approximately: ${\widehat{g_{N}}}_{n}^{[i]}=\hat{f}_{n}^{[i]}, n=0, \ldots, N+k, i=0,1$. With $g_{N}$ defined in this manner, it can be shown that the convergence rate does not deteriorate in comparison to polynomial subtraction [4].

The extension of this method to the $d$-variate cube is achieved by formulating an approximation $g_{N}$ satisfying

$$
{\widehat{g_{N}}}_{n}^{[i]}=\hat{f}_{n}^{[i]}, \quad 0 \leq n_{1}, \ldots, n_{d} \leq N+k, \quad i \in\{0,1\}^{d} .
$$

In this setting, $g_{N}$ consists of Cartesian products of univariate polynomials and modified Fourier eigenfunctions. Analysis of this approximation was presented in [1].

\subsection{The Fourier extension problem}

An alternative to the explicit subtraction of a smooth function interpolating boundary conditions is to enlarge the initial function space. A particular example that leads to interesting results and analysis is to consider both Laplace-Neumann and Laplace-Dirichlet eigenfunctions, as pursued in [12]. This combination of two orthogonal bases is no longer a basis itself, but a frame. It is overcomplete and therefore many representations of $f$ may exist. The approach taken in [12] to single out a useful representation is through a least squares criterion, following earlier results in $[6,7]$. It is shown that exponential convergence is achieved when $f$ is analytic.

Laplace-Dirichlet eigenfunctions on $[-1,1]$ are $\cos \left(n-\frac{1}{2}\right) \pi x$ and $\sin n \pi x, n=$ $1,2, \ldots$. Combined with (3) and rearranging terms, this leads to an approximation $g_{N}(x)$ of the form

$$
f(x) \approx g_{N}(x)=\frac{1}{2} a_{0}+\sum_{n=1}^{N}\left(a_{n} \cos n \frac{\pi}{2} x+b_{n} \sin n \frac{\pi}{2} x\right) .
$$

Thus one is looking for a periodic function on $[-2,2]$, to represent $f$ on $[-1,1]$. This function has exactly the form of a classical Fourier series, hence the name Fourier extension. It is shown in [12] that the least squares problem can be converted into a polynomial approximation problem. Convergence theory and fast algorithms then follow from existing results on orthogonal polynomials. In particular, assuming sufficient analyticity of $f$ near $[-1,1]$, we have (see [12, Th3.14])

$$
f(x)-g_{N}(x) \sim(3+2 \sqrt{2})^{-N}, \quad x \in[-1,1] .
$$

The extension of this approach to multivariate functions is straightforward in practice (see [7]), but delicate in theory. The generalisation of the theoretical analysis in [12] to many dimensions is a topic of current research. Preliminary results are along the lines of the theory described in Section 5. 
In Figure 1 we compare this approach and Eckhoff's method for univariate and bivariate examples. Exponential convergence of the former is verified. Despite offering only algebraic convergence, when $k=8$ Eckhoff's approach yields similar results.

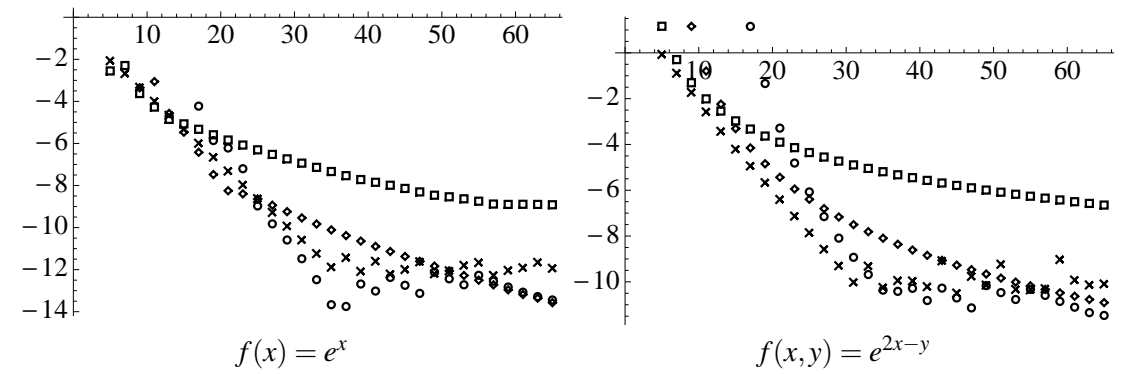

Fig. $1 \mathrm{Log}$ uniform error $\log _{10}\left\|f-g_{N}\right\|_{\infty}$ where $g_{N}$ is Eckhoff's approximation with $k=2$ (squares), $k=4$ (diamonds), $k=8$ (circles) or the Fourier extension approximation (crosses).

\section{The non-tensor product case}

The identification of modified Fourier expansions with eigenfunctions of the Laplace operator provides a useful link with geometry. The scope of multivariate modified Fourier expansions can be extended to include all domains for which eigenfunctions of the Laplace operator are known explicitly. A large and interesting family of domains consists of the so-called fundamental regions of root systems. These are simplical domains with special symmetry properties such that $\mathbb{R}^{d}$ can be tiled by reflecting the domain across all its sides and repeating the process indefinitely. It was first observed in [5] that eigenfunctions of the Laplace operator are obtained by symmetrizing classical multivariate Fourier series with respect to the set of symmetries described by a root system. The simplest example is the one-dimensional case, related to even symmetry in the root system $A_{1}$ :

$$
\cos n \pi x=\frac{1}{2} e^{i n \pi x}+\frac{1}{2} e^{-i n \pi x} .
$$

In two dimensions, three triangles are associated to root systems: the equilateral triangle (root system $A_{2}$ ), the right isosceles triangle $\left(B_{2}\right)$ and the triangle with angles $\frac{\pi}{2}, \frac{\pi}{3}$ and $\frac{\pi}{6}\left(C_{2}\right)$. The case of the equilateral triangle has been described in the context of modified Fourier series in more detail in [14]. In this case, each eigenfunction is a linear combination of six plane waves, and the symmetries involved are those of the dihedral group $D_{3}$. It is expected that the other cases can be treated 
similarly and, moreover, the theory of root systems may enable the approximation of multivariate functions on a long list of three- and higher dimensional simplices.

\section{References}

1. Adcock, B.: Convergence acceleration of modified Fourier series in one or more dimensions, Math. Comp. (to appear)

2. Adcock, B.: Multivariate modified Fourier series and application to boundary value problems, Num. Math. (to appear)

3. Adcock, B: Univariate modified Fourier methods for second order boundary value problems, BIT, 49, 249-280 (2009)

4. Barkhudaryan, A., Barkhudaryan, R. and Poghosyan, A.: Asymptotic behavior of Eckhoffs method for Fourier series convergence acceleration, Anal. Theory Appl., 23, 228-242, (2007)

5. Bérard, P.: Spectres et groupes cristallographiques I: domaines euclidiens, Inv. Math., 58, 179-199 (1980)

6. Boyd, J.: A comparison of numerical algorithms for Fourier extension of the first, second and third kinds, J. Comp. Phys.. 178, 118-160 (2002)

7. Bruno, O., Han, Y. and Pohlman, M.: Accurate high-order representation of complex threedimensional surfaces via Fourier continuation analysis, J. Comp. Phys., 227, 1094-1125 (2007)

8. Brunner, H., Iserles, A. and Nørsett, S. P.: The computation of the spectra of highly oscillatory Fredholm integral operators, J. Int. Eqn Appl. (to appear)

9. Bungartz, H.-J. and Griebel, M.: Sparse grids, Acta Numerica, 13, 147-269 (2004)

10. Eckhoff, K. S.: On a high order numerical method for functions with singularities. Math. Comp., 67, 1063-1087, (1998)

11. Evans, L. C.: Partial Differential Equations, AMS (1998)

12. Huybrechs, D.: On the Fourier extension of non-periodic functions, SIAM J. Numer. Anal. (to appear)

13. Huybrechs, D., Iserles, A. and Nørsett, S. P.: From high oscillation to rapid approximation IV: Accelerating convergence, IMA J. Num. Anal. (to appear)

14. Huybrechs, D., Iserles, A. and Nørsett, S. P.: From high oscillation to rapid approximation V: The equilateral triangle, Technical Report NA2009/04, DAMTP, University of Cambridge (2009)

15. Huybrechs, D. and Olver, S.: Rapid function approximation by modified Fourier series. In B. Engquist, A. Fokas, E. Hairer, and A. Iserles, editors, Highly Oscillatory Problems, pages 51-72, Cambridge, 2009, CUP

16. Iserles, A. and Nørsett, S. P.: From high oscillation to rapid approximation I: Modified Fourier expansions, IMA J. Num. Anal., 28, 862-887 (2008)

17. Iserles, A. and Nørsett, S. P.: From high oscillation to rapid approximation II: Expansions in polyharmonic eigenfunctions, Technical Report NA2006/07, DAMTP, University of Cambridge (2006)

18. Iserles, A. and Nørsett, S. P.: From high oscillation to rapid approximation III: Multivariate expansions, IMA J. Num. Anal. 29, 882-916 (2009)

19. Lanczos, C.: Discourse on Fourier series, Hafner, New York, (1966)

20. Lyness, J. N.: Computational techniques based on the Lanczos representation, Math. Comp., 28, 81-123, (1974)

21. Olver, S.: On the convergence rate of a modified Fourier series, Math. Comp. 78, 862-887 (2008)

22. Schmeißer, H.-J. and Triebel, H.: Topics in Fourier analysis and function spaces, Wiley (1987)

23. Temlyakov, V.: Approximation of Periodic Functions, Nova Sci., New York (1993) 